\title{
BEWEIS DES SATZES
}

DASS EINE JEDE

\section{ALGEBRAISCHE GLEICHUNG EINE WURZEL HAT}

\author{
VON \\ ELLING HOLST \\ in CHRISTIANIA.
}

Unserem Zwecke ist offenbar Genüge gethan, wenn wir folgenden Satz beweisen:

Wenn eine jede ganze Function des $(n-\mathrm{I})^{\text {ten }}$ Grades sich in $n-\mathrm{I}$ lineare Factoren auflösen lässt, so hat eine Gleichung des $n^{\text {ten }}$ Grades wenigstens eine Wurzel.

Die Gleichung, in welcher der Einfachheit wegen dem Coefficienten von $x^{n}$ der Werth = I beigelegt sein mag, lässt sich immer so schreiben:

$$
x f(x)=K
$$

wo $f(x)$ vom $(n-\mathrm{I})^{\text {ten }}$ Grade ist, und also nach unserer Voraussetzung sich in die Factoren $\left(x-\alpha_{1}\right)\left(x-\alpha_{2}\right) \ldots\left(x-\alpha_{n-1}\right)$ auflösen lässt. Da mehrere der Grössen $\alpha_{i}$ einander gleich sein und einige derselben auch $=\mathrm{o}$ sein können, so ergiebt sich als allgemeinste Form der Gleichung:

$$
x^{m_{0}}\left(x-\alpha_{1}\right)^{m_{1}} \ldots\left(x-\alpha_{p}\right)^{m_{p}}=K,
$$

wo

$$
m_{0}+m_{1}+\ldots+m_{p}=n
$$

Nun setzen wir:

$$
x=r_{0} e^{i \varphi_{0}}, \quad x-\alpha_{j}=r_{j} e^{i \varphi_{j}}, \quad K=R e^{i \phi} ;
$$


dann zerfüllt die Gleichung ( $\mathrm{I}$ ) in die beiden Gleichungen: die Modulgleichung:

$$
r_{0}^{m_{m_{1}}} r_{1}^{m_{1}} \ldots r_{p}^{m_{p}}=R
$$

und die Argumentgleichung:

$$
m_{0} \varphi_{0}+m_{1} \varphi_{1}+\ldots+m_{p} \varphi_{p}=\phi+2 k \pi
$$

wo man die $\varphi_{0}, \varphi_{1}, \ldots, \varphi_{p}$, so wie die $\Phi \overline{\overline{>}}$ o und $<2 \pi$ annehmen darf, während $k$ jede beliebige ganze positive oder negative Zahl oder Null vorstellt.

Sei nun ferner $P$ ein laufender Punkt in der Gaussischen $(x+i y)$ Ebene, $A_{0}$ der Nullpunkt des letzteren, $A_{1}, \ldots, A_{p}$ die den Complexen $\alpha_{1}, \ldots, \alpha_{p}$ entsprechenden Punkte, so werden wir zunächst die Grösse

$$
P A_{0}^{2 m_{2}} P A_{1}^{2 m_{1}} \ldots P A_{p}^{2 m_{p}}-R^{2}=\Psi(x, y)
$$

zu untersuchen haben, wo $x$ und $y$ jetzt als gewöhnliche Cartesische Coordinaten aufzufassen sind, und $\psi(x, y)$ eine ganze Function von $x$ und $y$ vom Grade $2 n$ vorstellt.

Verlegt man $P$ in einen der Punkte $A_{0}, A_{1}, \ldots, A_{p}$, so wird:

$$
\Psi(x, y)<0 .
$$

Beschreibt man um einen willkürlichen Mittelpunkt $C$ einen Kreis mit dem Radius $=m+\sqrt[i]{R}+\varepsilon$, wo unter $\varepsilon$ eine willkürliche positive Grösse zu verstehen ist, und $m$ den grössten unter den Abständen des Punktes $C$ von einem der Punkte $A_{0}, A_{1}, \ldots, A_{p}$ vorstellt, so liegt jeder der letztgenannten Punkte innerhalb dieses Kreises, und die Abstände aller dieser Punkte von jedem in der Peripherie des Kreises liegenden Punkt $Q$ sind immer grösser als $\sqrt[n]{R}$. Legt man daher $P$ in irgend welchen derartigen Punkt $Q$, so erhält man:

$$
\Psi(x, y)>0
$$

Aus diesen beiden Ungleichheiten ergiebt sich, auf Grund der Continuität der ganzen Function $\Psi$ für jede reelle und endliche Variation von $x$ und $y$, der Schluss, dass jede beliebige continuirliche Verbindungskurve zwischen dem laufenden Punkt $Q$ des Kreises und irgend einem der mehrerwähnten 
Beweis des Satzes dass eine jede algebraische Gleichung eine Wurzel hat.

Punkte, z. B. dem Punkte $A_{0}$, zum mindesten einen Punkt enthalten muss, wo

$$
\Psi(x, y)=0
$$

Ls besteht demgemäss eine stetige, geschlossene Contour rings um $A_{0}$, in welcher die Modulbedingung (3) überall erfüllt ist.

Wenn die hier gefundene Contour nicht noch andere jener Punkte, ausser allein $A_{0}$, umschliesst, so werden jene übrigen Punkte, jeder für sich allein oder mehrere zusammen, von ähnlichen Contouren umgeben sein. Aus dem Umstande, dass $A_{0}$ auf einem endlich und stetig begrenzten Theil der Ebene liegt, folgt nun aber jedenfalls, dass der Vectorradius $A P$, wenn $P$ den ganzen Umfang dieser Begrenzung einmal durchwandert, einen Umlauf von der Grösse $\pm 2 \pi$ ausgeführt hat. Das Zeichen hängt $\mathrm{ab}$ von der Richtung, in welcher der Umlauf bewerkstelligt ist, und bleibt dasselbe, wo innerhalb der Contour der Punkt $A_{0}$ auch liegen mag.

Wir nehmen an, dass die Punkte:

$$
\left.A_{0}, A_{1}, \ldots, A_{q}, \quad 0 \leqq q<p\right)
$$

innerhalb der gleichen Contour liegen, und dass somit die übrigen $p-q$ Punkte sich ausserhalb der Contour befinden. Wird nun letztere von einem Anfangspunkt $P_{0}$ aus einmal in positiver Richtung durchlaufen, so werden die Argumente, welche den von den inneren Punkten ausgehenden Vectorradien entsprechen, je um $2 \pi$ gewachsen sein, während diejenigen, welche den von den äusseren Punkten ausgehenden Vectorradien entsprechen, nach einem gewissen Oscilliren alle wieder zu ihrem Anfangswerth zurückgekehrt sein werden. Werden nun alle diese Argumente, die allen den genannten Vectorradien entsprechen und deren Werthe z. B. beim Anfang des Umlaufs $\overline{>}$ o und $<2 \pi$ angesetzt werden können, in das erste Glied der Argumentgleichung (4) eingesetzt, so wird während des Umlaufes dieses Glied continuirlich wachsen um die Grösse:

$$
\left(m_{0}+m_{1}+\ldots+m_{q}\right) 2 \pi
$$

d. h. die Contour enthält $m_{0}+m_{1}+\ldots+m_{q}$ Punkte, welche der Argumentbedingung genügen. Der kleinste Werth, den diese Zahl annehmen kann, ist I (nämlich für $m_{0}=\mathrm{I}, q=0$ ). Also hat die Gleichung wenigstens eine Wurzel; was zu beweisen war. 
Durch Betrachtungen von ganz analoger Art, wie die eben angestellten, wobei man nur noch zu berücksichtigen hat, dass die Anzahl der Wurzeln, oder, genauer gesprochen, die Anzahl der linearen Factoren $n$ nicht überschreiten kann, wird man noch ohne Schwierigkeit die Richtigkeit folgender Sätze einsehen:

I. Während immerhin innerhalb einer Contour mehr als eine Wurzel liegen kann, kann umgekehrt um einen der Punkte $A_{0}, A_{1}, \ldots, A_{p}$ nicht mehr als eine Contour verlaufen.

2. Die Anzahl der Wurzeln auf jeder Contour ist immer gleich der Summe der Multiplicitäten der eingeschlossenen A-Punkte.

3. Diese Wurzelpunkte theilen die Contour in Bogen, welche von sämtlichen Wurzelpunkten nach derselben Richtung durchlaufen werden, wenn $\Phi$, das Argument von $K$, um $2 \pi$ wächst. Unter diesen Umständen findet also nur eine circulare Vertauschung der Wurzeln auf derselben Contour statt.

4. Eine Doppelwurzel hat die Gleichung nur dann, wenn die Modulkurve $\Psi(x, y)=0$ einen gewöhnlichen Knotenpunkt besitzt, in welchem zwei Contouren zu einer sich selbst schneidenden Contour vereinigt sind. Für einen bestimmten Werth von $\Phi$ werden dann die beiden Wurzeln der beiden Contoure unter dem gleichartigen Umlauf im Knotenpunkte zusammentreffen.

In ganz ähnlicher Weise hätte die ursprüngliche Gleichung auch folgende Gestalt haben können:

$$
x^{t} f(x)=\varphi(x), .
$$

wo $f(x)$ vom $(n-t)^{\text {ten }}$, und $\varphi(x)$ vom $(t-1)^{\text {ten }}$ Grad ist. Nehmen wir an, dass hier die Auflösbarkeit der ganzen Function in lineare Factoren für die grössere der beiden Gradzahlen $n-t$ und $t-\mathrm{I}$ (somit aber auch für die kleinere) oder, wenn $n-t=t-\mathrm{I}$, für diese Gradzahl nachgewiesen ist, so lässt die Gleichung sich schreiben:

$$
x^{m_{0}}\left(x-\alpha_{1}\right)^{m_{1}} \ldots\left(x-\alpha_{p}\right)^{m_{p}}=K\left(x-\beta_{1}\right)^{n_{1}} \ldots\left(x-\beta_{q}\right)^{n_{4}},
$$

wo

$$
m_{0}+m_{1}+\ldots+m_{p}=n \text { und } n_{1}+n_{2}+\ldots+n_{q}=t-\mathrm{r} .
$$


Man erhält dann:

$$
\imath_{0}^{m_{0}} \cdot r_{1}^{m_{1}} \ldots r_{p}^{m_{p}}=R s_{1}^{n_{1}} \ldots s_{q}^{n_{q}}
$$

und

$$
m_{0} \varphi_{0}+m_{1} \varphi_{1}+\ldots+m_{p} \varphi_{p}=\phi+2 k \pi+n_{1} \phi_{1}+\ldots+n_{q} \psi_{q} .
$$

Die Kurve:

$$
P A_{0}^{2 m_{0}} \ldots P A_{p}^{2 m_{p}}=R^{2} \cdot P B_{1}^{2 n_{1}} \ldots P B_{q}^{2 n_{q}},
$$

(wo $A_{0}, \ldots, A_{p}$, dieselbe Bedeutung haben, wie oben, während $B_{1}, \ldots, B_{q}$ in ahnlicher Weise den Wurzeln $\beta_{0}, \ldots, \beta_{q}$ entsprechen) besteht jetzt, wic man alsbald übersieht, aus:

I) einer oder mehreren $A$-Contouren, die sämtliche $A_{i}$ umschliessen, doch so, dass keiner von mehr als einer Contour umgeben ist, und

2) einer oder mehreren B-Contouren, welche in dem Fall, wo irgend ein $B_{i}$ von irgend einer $A$-Contour umschlossen wird, Enclaven innerhalb der $A$-Contouren bilden und jenes $B_{i}$ in solcher Weise umgeben, dass alle $A$-Punkte von allen $B$.Punkten geschieden sind. Kein $B$-Punkt liegt innerhalb mehr als einer $B$-Contour. Die ausserhalb der $A$-Contouren liegenden Punkte $B_{i}$ werden nicht von Contouren umschlossen.

Die gesammte Multiplicitat der in den Enclaven eingeschlossenen Punkte $B_{i}$ betrage $t^{\prime}$, wo also

$$
\mathrm{O} \overline{\overline{<}} t^{\prime} \leqq t-\mathrm{I} \text {. }
$$

Beim Umlauf durch alle Contouren gehören dann $n-t^{\prime}$ Wurzeln den $A$-Kurven die übrigen $t^{\prime}$ den $B$-Kurven. Das Raisonnement ist ein ganz. analoges, wie bei dem vorigen Fall, der ja auch nur einen Unterfall des hicr behandelten darstellt, bei welchem $t=1$, d. h. $t^{\prime}=0$ geworden ist.

Besonders beachtenswerth ist der Fall

$$
n-t=t-\mathrm{I}, \text { d. h. } n=2 t-\mathrm{I} \text {. }
$$

Man schliesst hier, dass der Satz für die Gradzahl $2 t$ - I gilt, weil er für die Gradzahl $t$ - I gilt und erweitert denselben in solcher Weise rasch von dem $4^{\text {ten }}$ Grad auf den $9^{\text {ten }}$, den I $9^{\text {ten }}$, den $39^{\text {ten }}$ Grad u. s. w. 
Die verschiedenen Systeme der Contouren, auf welchen die Wurzeln nach dem hier gesagten sümmtlich liegen müssen, lassen sich, wenn es sich nicht um den Beweis für die Existenz der Gleichungswurzel handelt, ins Unendliche vermehren.

Christiania, I 5 Jan. 1886. 